LA W RENCE LIVERMORE NATIONAL LABORATORY

\title{
A General Dead-Zone Predicted With The Detonation Shock Dynamics
}

J. Yao

July 1, 2013 
This document was prepared as an account of work sponsored by an agency of the United States government. Neither the United States government nor Lawrence Livermore National Security, LLC, nor any of their employees makes any warranty, expressed or implied, or assumes any legal liability or responsibility for the accuracy, completeness, or usefulness of any information, apparatus, product, or process disclosed, or represents that its use would not infringe privately owned rights. Reference herein to any specific commercial product, process, or service by trade name, trademark, manufacturer, or otherwise does not necessarily constitute or imply its endorsement, recommendation, or favoring by the United States government or Lawrence Livermore National Security, LLC. The views and opinions of authors expressed herein do not necessarily state or reflect those of the United States government or Lawrence Livermore National Security, LLC, and shall not be used for advertising or product endorsement purposes.

This work performed under the auspices of the U.S. Department of Energy by Lawrence Livermore National Laboratory under Contract DE-AC52-07NA27344. 


\title{
A General Dead-Zone Predicted With The Detonation Shock Dynamics
}

\author{
Yao, Jin \\ Lawrence Livermore National Laboratory, California, USA
}

\begin{abstract}
Experiments and direct numerical simulations show that a dead-zone may occur after a detonation makes a turn at a corner $[1],[7],[8],[9],[10],[13],[14]$, thus to affect the propagation of a lighting front. However, whether or not a dead-zone still exists when the curvature of the obstacle is reduced seems not yet answered. This report applies the detonation shock dynamics (DSD) theory with the consideration that the DSD solution is a perturbation to a Huygens Construction, and predicts that a dead-zone would always exist mathematically when a detonation makes a turn, even with a smooth obstacle.

The physical size of a dead-zone in the case of a corner-turning is derived to be the same as the critical radius based on the DSD studies ${ }^{[2],[4],[5]}$ i.e. the inverse of the critical (failure) curvature at the right most turning point on a $D_{n}-\kappa$ curve. In the case the radius of curvature of the boundary of the obstacle is much larger than the critical radius, the thickness of the dead-zone is thin because it is mathematically of a higher order, thus it may hardly be observed in experiments.

A marked particle DSD wave-tracker is employed to verify the fact that the detonation velocity on the surface of an smooth obstacle is reduced by the $D_{n}-\kappa$ curvature law and the DSD boundary angle to be lower than the failure value. This gives support to the conclusion that a general dead zone exists when a detonation turns over an arbitrary obstacle. The marked particle DSD wave-tracker is verified with an analytical integral of the system for a rate-stick geometry. The tracker is second order convergent in space. A burntable for PBX-9502 is created with the DSD tracker, and a local quadratic least-squared fitting converts the data of the burn-table to lighting times on the nodes of a hydro mesh.

It is expected that with a careful treatment for a $D_{n}-\kappa$ relation with a critical failure curvature, the dead-zone predicted in this report can be simulated with the marked particle DSD wave-tracker we have.
\end{abstract}




\section{INTRODUCTION}

A lighting time calculation for HE materials for a hydro simulation is important for a correct predication of energy release. Experiments and direct numerical simulations show that the velocity of propagation of a detonation front is affected by the front curvature. In the case of a sufficiently high positive front curvature (diverging wave), the detonation can be quenched. In the case of a corner-turning, a locally highly curved front forms when the detonation makes a turn over the corner. An inactive (or slowly reacting) region, the so-called dead-zone can be formed there. The above behaviors can be described with the theory of detonation shock dynamics (DSD).

The detonation shock dynamics (DSD) theory can be used to derive a set of evolution equations for a quasi-steady detonation propagation in an unbounded homogeneous HE media. These front evolution equations predict that the front velocity of a weakly curved detonation is affected by the front curvature to the leading order. It is to say that

$$
D_{n}=f(\kappa)
$$

where $D_{n}$ is the detonation normal velocity and $\kappa$ is the front curvature.

When the front is flat, the front velocity is a constant and is noted by $D_{C J}$ which is the Chapman-Jouguet velocity of a ZND wave. A positive front curvature (for a diverging wave) will slow the detonation down. In the case of a corner-turning, the curvature would be positive, and such a $D_{n}-\kappa$ relation can be used to describe the interior front propagation. Near a (confined) boundary, the normal of a detonation would not be affected by the boundary if the angle $\omega$ between front and the boundary is smaller than a given angle $\omega_{c}$. Otherwise a dragging occurs and the normal is bent toward boundary to keep $\omega=\omega_{c}{ }^{[3]}$.

A dead-zone may occur when a detonation makes a turn on the boundary where front curvature exceed the critical value and cause the detonation propagation to fail. This report will discuss a dead-zone predicted naturally with the DSD theory, that can explain numerical and experimental results to certain extent.

\section{AN ANALYTICAL HUYGENS SOLUTION OVER A 2D OBSTACLE}

In figure 1, a point lighting source $S$ at $\left(x_{S}, y_{S}\right)$ and an obstacle defined by $y=f(x)$ as its boundary are shown. For the discussion in this report, we assume $f^{\prime \prime}(x)$ does not change sign so there is no turning points on the boundary of the obstacle. The 


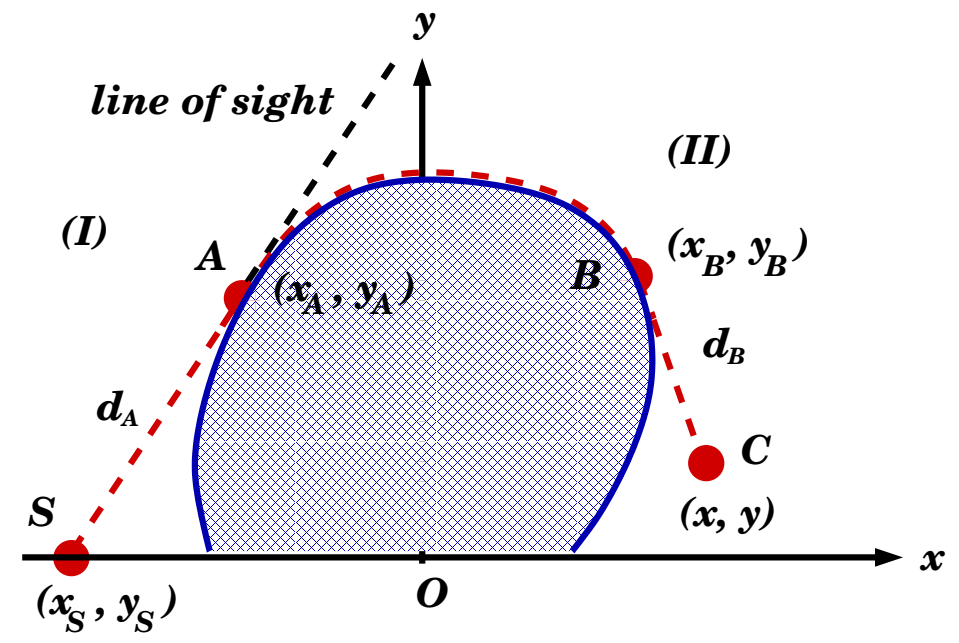

Figure 1: The path of lighting (a characteristic line, colored with red) over an obstacle (with boundary colored blue) for a point $C$ behind the line of sight. $S$ is the source of lighting. $A$ and $B$ are tangential points.

solution of a Huygens Construction at point $C$ with $\vec{r}_{C}=(x, y)$ is the minimum distance from $S$ to $C$. Assume there is no turning point existing on the boundary of the obstacle (this is often the case), then the solution can be directly written as

$$
D(x, y)=d_{A}+\int_{A}^{B} \sqrt{1+\left[f^{\prime}(\xi)\right]^{2}} d \xi+d_{B}
$$

The point $A$ and $B$ make the tangential lines $d_{A}=|\overline{\mathbf{S A}}|$ and $d_{B}=|\overline{\mathbf{B C}}|$. A general tangent point at $B$ (i.e., the $\left(x_{B}, y_{B}\right)$ in fig. 1 ) associated with a point $(x, y)$ can be computed with the analytical equation

$$
f\left(x_{B}\right)-y=f^{\prime}\left(x_{B}\right)\left(x_{B}-x\right) .
$$

\section{AN EXPLICIT SOLUTION FOR A BOUNDARY DESCRIBED BY AN ELEMENTARY CURVE}

Explicit solutions of the tangential points ( $A$ and $B$ in fig. 1$)$ may exist when the functional form of $f(x)$ is simple enough. For example, in the case that the obstacle 
is represented with an elliptic

$$
\frac{x^{2}}{a^{2}}+\frac{y^{2}}{b^{2}}=1,
$$

the solution of the tangential point $\left(x_{T}, y_{T}\right)$ associated with a given point $\left(x^{*}, y^{*}\right)$ is

$$
\begin{gathered}
x_{T}=\frac{a^{2} b^{2} x^{*} \pm a^{2} y^{*} \sqrt{a^{2}\left(y^{*}\right)^{2}+b^{2}\left(x^{*}\right)^{2}-a^{2} b^{2}}}{a^{2}\left(y^{*}\right)^{2}+b^{2}\left(x^{*}\right)^{2}}, \\
y_{T}= \pm b \sqrt{1-\frac{x_{T}^{2}}{a^{2}}} .
\end{gathered}
$$

In the case that the source $\left(x_{S}, y_{S}\right)$ is on the $x$-axis, that $y_{S}=0$, the solution is simplified to

$$
x_{A}=\frac{a^{2}}{x_{S}}, \text { and } y_{A}= \pm b \sqrt{1-a^{2} / x_{S}^{2}},
$$

then the whole solution becomes

$$
D(x, y)=d_{A}+\int_{\theta_{a}}^{\theta_{b}} \sqrt{a^{2} \cos ^{2}(\theta)+b^{2} \sin ^{2}(\theta)} d \theta+d_{B}(x),
$$

where

$$
\begin{gathered}
\theta_{a}=\arctan \left(\frac{y_{A}}{x_{A}}\right), \theta_{b}=\arctan \left(\frac{y_{B}}{x_{B}}\right), \\
d_{A}=\sqrt{\left(\frac{a^{2}}{x_{0}}-x_{0}\right)^{2}+b^{2}\left(1-\frac{a^{2}}{x_{0}^{2}}\right),} \\
d_{B}(x)=\sqrt{\left(x-x_{B}\right)^{2}+\left(y-y_{B}\right)^{2}},
\end{gathered}
$$

where $\left(x_{B}, y_{B}\right)$ is the tangential point on the obstacle associated with $(x, y)$, defined in explicitly given by (eq. 3). The term in the middle for the arc-length is an elliptic integral in this case. Without loss of generality, we assume that $y \geq 0$ in the above case because of symmetry.

\section{THE GEOMETRY OF A HUYGENS FRONT NEAR AN OBSTACLE}

In the case of propagating with a constant velocity 1 , the front geometry at time $t$ can be defined by the distance function $D(x, y)$

$$
\phi(x, y ; t)=D(x, y)-t=0 .
$$


The normal direction of the front is determined by

$$
\vec{\nabla} \phi=D_{x} \hat{\imath}+D_{y} \hat{\jmath}
$$

$D_{x}, D_{y}$ are derivatives of the distance function $D(x, y), \hat{\imath}$ and $\hat{\jmath}$ are the directional unit vectors. The normal vector $\hat{n}$ is defined by $\vec{\nabla} \phi /|\nabla \phi|$ from differential geometry. By definition, $D(x, y)$ is the solution of the Eikonal equation $|\nabla D(x, y)|=1$, therefore $|\vec{\nabla} \phi| \equiv 1$, the normal is simply $\hat{n}=D_{x} \hat{\imath}+D_{y} \hat{\jmath}$.

If interested, differentiating (eq. 2) very carefully with the consideration that $x_{B}=x_{B}(x, y)$ and applying (eq. 3) would provide

$$
D_{x}=\frac{x-x_{B}}{d_{B}}, D_{y}=\frac{y-y_{B}}{d_{B}}
$$

where $d_{B}=\sqrt{\left(x-x_{B}\right)^{2}+\left(y-y_{B}\right)^{2}}$.

Then the front curvature can be computed by $\kappa=\vec{\nabla} \cdot \hat{n}=\nabla^{2} D(x, y)=D_{x x}+$ $D_{y y}$. A careful evaluation gives

$$
D_{x x}=\frac{1}{d_{B}}-\frac{\left(x-x_{B}\right)^{2}}{d_{B}^{3}}, D_{y y}=\frac{1}{d_{B}}-\frac{\left(y-y_{B}\right)^{2}}{d_{B}^{3}},
$$

therefore

$$
\kappa=\nabla^{2} D(x, y)=\frac{1}{d_{B}} .
$$

A fact that may not be visually noticeable but is mathematically obvious, is that when point $C$ is close to the boundary of the obstacle, $d_{B}$ can become arbitrarily small, thus to make the front curvature approach positive infinity.

\section{A PHYSICAL EXPLANATION}

It may be a bit of a surprise at first that from (eq. 8) through (eq. 10), the radius of the front curvature can be seen as the distance from a point $C$ at $(x, y)$ behind the obstacle to the tangential point $B$ on the obstacle associated with $C$ (figure 2 ). However, if one carefully inspects the expressions of $D_{x}, D_{y}$, and the curvature $\kappa=\nabla^{2} D(x, y)$ in equations (9) and (10), it becomes clear that the solution of the distance function over an obstacle is the same as a direct distance measurement as if the lighting source is set at the tangential point $\left(x_{B}, y_{B}\right)$, because the tangential point has the shortest distance to travel to $(x, y)$. Then the front curvature $\kappa$ at 


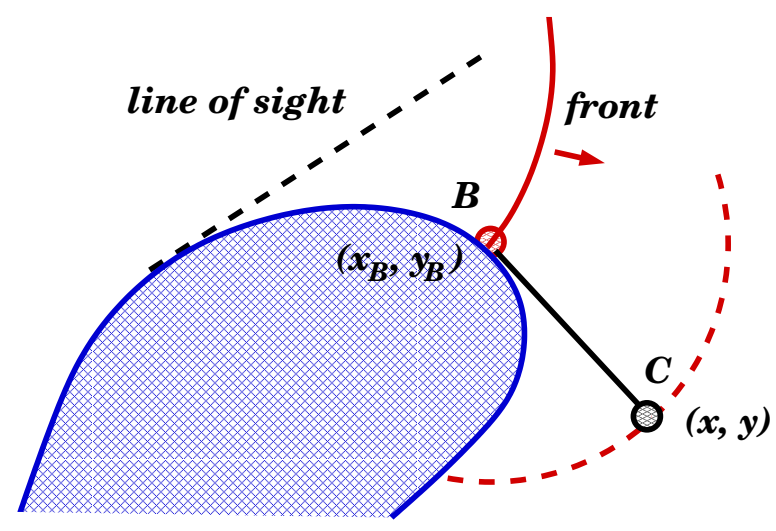

Figure 2: The tangential point $B$ as a new lighting source for a point $C$ in the tangential line. Locally the lighting front, when passing $C$, is a piece of the circle (dash red curve) centered at $B$ in the neighborhood of $C$.

$(x, y)$, must be $d_{B}^{-1}$, the inverse of the radius of the circular front centered at $\left(x_{B}, y_{B}\right)$. $\kappa$ becomes infinity naturally as $d_{B}$ goes to zero.

The above argument is solely based on the observation that, if a dead zone does not exist when the detonation makes a turn over an obstacle, the intersection $\left(x_{B}, y_{B}\right)$ of front and the boundary of the obstacle would be physically a new lighting source to any point on the tangential line (which is a characteristic line in this problem), because the tangential line is the shortest-path solution. Then a contradiction occurs because the front curvature (the inverse of the radius of the "detonationsphere" centered at the tangential point) would be too big for the detonation to travel to any potion on the tangential line sufficiently close to the tangential point . Therefore a dead zone (as a boundary layer) has to exist when a detonation makes a turn over the obstacle.

\section{THE DSD PREDICTION OF A DEAD-ZONE OVER AN OBSTACLE}

For a quasi steady detonation described with the detonation shock dynamics (DSD), the normal velocity $D_{n}$ of the shock front is a function of the front curvature $\kappa$. In the most general case, for a HE material of a large activation energy, the sub-sonic 

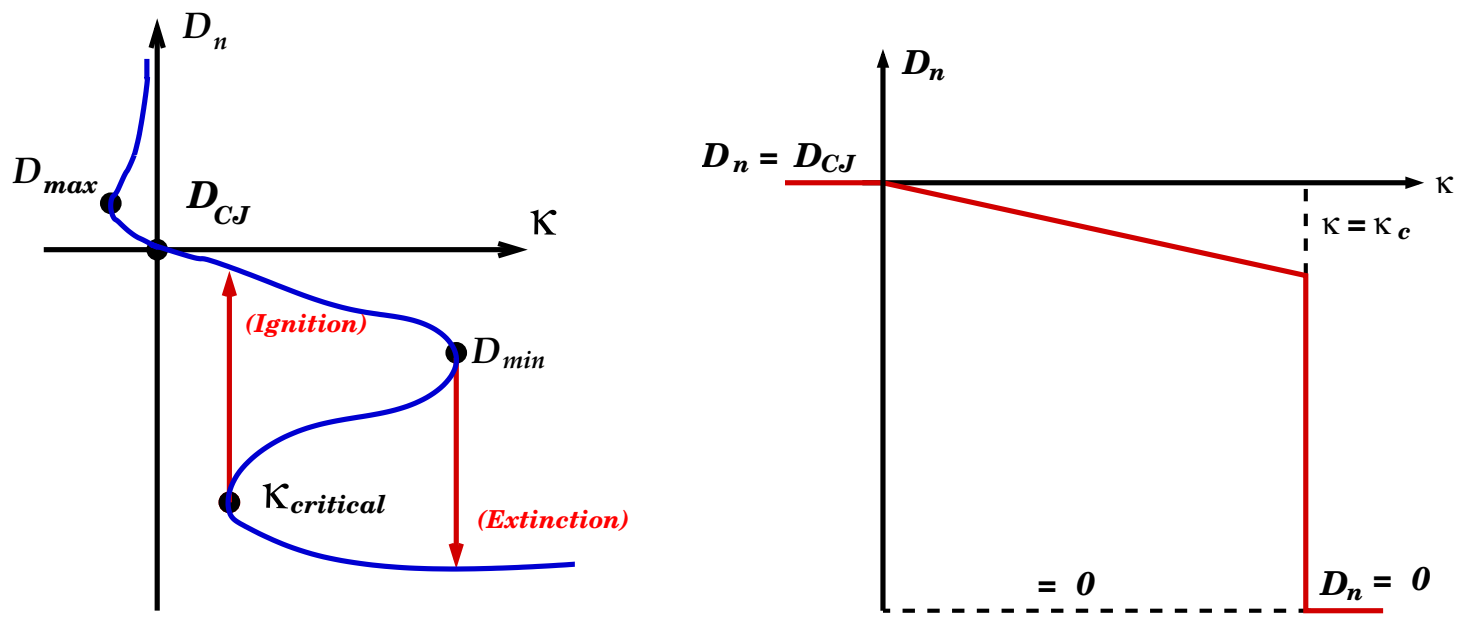

Figure 3: Left: a typical $\mathbf{D}_{\mathbf{n}}-\kappa$ curve with turning points obtained with the detonation shock dynamics. Right: a piece-wise linear $\mathbf{D}_{\mathbf{n}}-\kappa$ employed for the discussion in this paper.

portion (for a diverging detonation front propagation) of a $D_{n}-\kappa$ curvature looks like a backward $S$ as shown in figure 3. A couple of turning points always exist. No steady detonation can occur for a radius of curvature smaller than $\kappa_{\text {max }}^{-1}$. This is to say for a point over the obstacle with a tangential distance to the obstacle (which defines the front curvature) less than $\kappa_{\max }^{-1}$, there will be no detonation because the front curvature would be too high for a steady detonation front to propagate further.

Because any given point on boundary of the obstacle can be considered as a lighting source in its neighborhood (for the region out of the line of sight) will for sure produce a front curvature greater than the critical value assuming the detonation can keep going from there. Therefore the detonation must die there. Only when the front curvature is smaller than $\kappa_{\max }$ (the tangential distance is greater than $R_{c}$ ), the propagation of the detonation is possible (figure 4). The above argument, which is a physically general one, does not depend on the solution of the Huygens construction (eq. 2) over an obstacle. However, the solution of the Huygens construction can be considered as the leading order approximation of a DSD solution, and can be used to describe the geometry of a dead-zone to the leading order. 


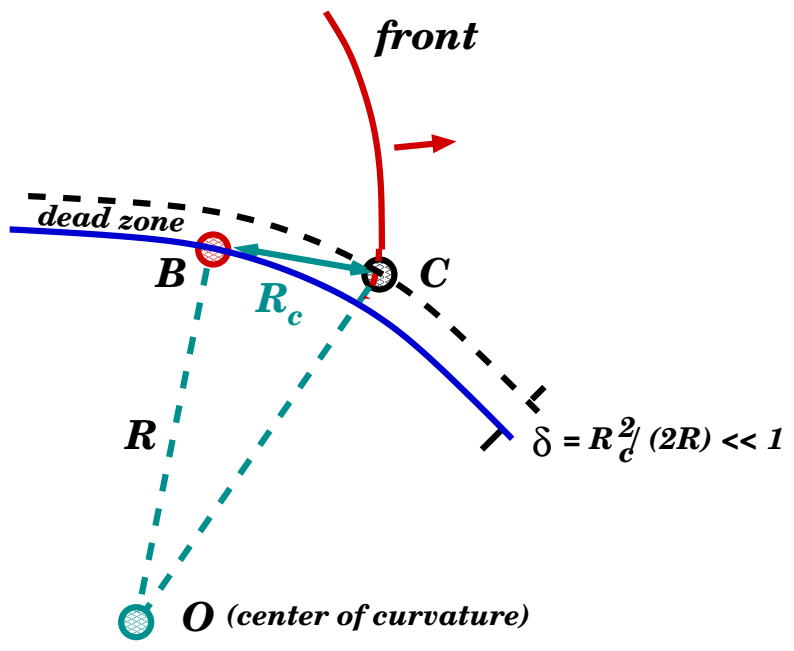

Figure 4: $A$ dead-zone starts to form at a point $C$ with a tangential distance of $R_{c}$ to the tangential point $B$, as the front curvature arrives at $\kappa_{\max }=R_{c}^{-1}$. The dashed line marks the boundary of a dead-zone bounded also by the the boundary of the obstacle (blue line). Note that $O C B$ is a right triangle.

\section{THE $D E A D-Z O N E$ FOR A CIRCULAR OBSTACLE}

The LANL technique report ${ }^{[15]}$ gives an analytical solution for a Huygens Construction for a circular obstacle. A circle is a special case of an elliptic curve with $a=b=R$, with $R$ the radius if the circle. Therefore the dead-zone is the region between the circular obstacle $x^{2}+y^{2}=R^{2}$ and the circle $x^{2}+y^{2}-R^{2}=R_{c}^{2}$, where $R_{c}=1 / \kappa_{\max }$ the radius for the critical curvature $\kappa_{\max }$. This dead-zone is bounded by the line of sight (figure 5).

The thickness of this dead-zone (see fig. 4) is $\sqrt{R^{2}+R_{c}^{2}}-R=R_{c}^{2} / 2 R$ plus higher order terms. In the case that $R_{c} / R=O(\epsilon) \ll 1$ (usually true for a smooth obstacle in real problems because the critical radius of curvature is much smaller than the dimension of the obstacle), the dead-zone thickness is of the order $\epsilon R_{c} / 2$, a small portion of a small length $R_{c}$. This explains why a dead-zone is hardly observed in experiments other than a corner-turning.

The radius of curvature of the detonation front for PBX-9502 is about $2(\mathrm{~cm})$ in an axi-symmetrical corner-turning calculation where the dead-zone arrives its maximum dimension ${ }^{[7]}$. Because in $3 D$ the curvature of a detonation sphere is twice as big as in $2 D$ for a given radius, the $2 D$ critical radius would be half of its $3 D$ size. 
Then the critical radius for this type of HE, as discussed in previous sections can be reasonably assumed to $R_{c} \sim 1(\mathrm{~cm})$ (the $2 D$ critical curvature). It is reasonable to set the radius of the curvature of the obstacle to be $R=10 R_{c} \sim 10(\mathrm{~cm})$ for it is about the dimension of the practical problem, then the corresponding thickness of the dead-zone is $\delta=R_{c}^{2} / 2 R=0.05 R_{c}=0.05(\mathrm{~cm})$. A thickness of this value is difficult to be observed. In fact it is probably much smaller than the size of a computational cell.

Cylinder tests also show that the failure diameters for LX-17 is about $1.1-$ $1.2(\mathrm{~cm})$, and $0.7-0.8(\mathrm{~cm})$ for PBX $9502^{[8]}$. If the DSD boundary angle is $\omega_{c}=\pi / 3^{[1]}$, the failure radius $R_{c}\left(\kappa_{\max }^{-1}\right)$ will be of the same values, i.e. about $1(\mathrm{~cm})$. At the detonation failure velocity (usually about ten percent reduction from the CJ value [13], for PBX 9501), for a linear $D_{n}-\kappa$ relation, it means $\left|\vec{r}-\vec{r}_{B}\right| / \alpha \sim 10$ at the edge of the dead-zone. $\alpha$ is then not a free parameter, rather a "material property". Its value should be the order of $0.1(\mathrm{~cm})$ for PBX-9501 because this value would reduce the detonation velocity by about 10 percent from the CJ value and quench the detonation at the critical radius $\sim 1(\mathrm{~cm})$.

Because of all the above, we can reasonably choose $R \sim 10(\mathrm{~cm}), R_{c} \sim 1(\mathrm{~cm})$, and $\alpha \sim 0.1(\mathrm{~cm})$ as a typical parameter set for later discussions.

\section{THE DEAD-ZONE IN A CORNER-TURNING PROBLEM}

In the case of a corner-turning (as shown in fig. 5), the tip of the corner $\left(x_{T}, y_{T}\right.$ ) becomes the new lighting source for an point that is not in the line of sight. It is the limit of $R \rightarrow 0$ in the above case of a circular obstacle. The "thickness" of the deadzone will be $R_{c}=\kappa_{\max }^{-1}$. Therefore, the dead-zone has the shape of a piece of a pie, with $\left(x_{T}, y_{T}\right)$ as the center, $\kappa_{\text {max }}^{-1}$ as the radius, bounded by the line of sight (defined by the initial lighting source and the tip of the corner), and the top boundary of the corner. An experimentally observed dead-zone may have its boundary shifted because the measurements take place a while after the corner-turning occurs. The area of this dead-zone is $\theta_{c} R_{c}^{2}$ to the leading order. The value of the DSD failure (critical) radius $R_{c} \sim 1(\mathrm{~cm})$ that we have chosen based on cylinder tests ${ }^{[8]}$ is just about the same size of the dead-zone observed in corner-turning experiments and direct numerical simulations ${ }^{[7],[9],[14]}$. This observation strongly supports the above result derived from the DSD theory. 


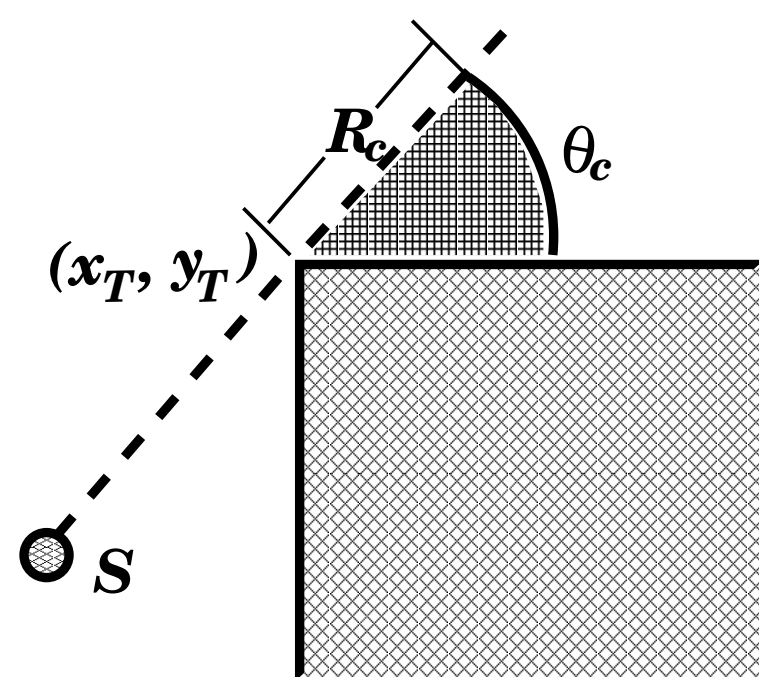

Figure 5: The leading order geometry of the dead-zone for a corner turning.

\section{THE DEAD-ZONE FOR AN GENERAL OBSTACLE}

The boundary of the dead-zone is defined by the path of the point with a tangential distance of the critical length to the boundary of the obstacle. Fig. 6 shows a deadzone for a general obstacle. Mathematically, any portion of a sufficiently smooth surface can be fit with a circle for a $3^{\text {rd }}$ order accuracy, therefore locally most results obtained previously for a circular obstacle still hold here.

The propagation of a steady detonation does not exactly follow the solution of a Huygence construction. Because of a positive front curvature $\kappa$ would slow down the front velocity $D-n$. The more curved the front, the slower the front velocity. This effect is described with a $D_{n}-\kappa$ relation derived from the detonation shock dynamics (DSD) ${ }^{[4]}$. On the boundary of a confined HE material, the angle $\omega$ between a detonation front and the confinement must be limited with a specific DSD angle $\omega_{c}$ between a detonation front and the wall ${ }^{[3]}$.

The mathematical analysis that has predicted a general dead-zone for a Huygens front turning over an obstacle can not be directly applied to a DSD front because the complication of the $D_{n}-\kappa$ relation and the boundary angle constraint. However with a numerical method to track the detonation front for a given $D_{n}-\kappa$ and a DSD boundary angle $\omega_{c}$, one is able to compute the terminal detonation velocity at the point the front meeting the boundary. If the terminal velocity is below the failure 


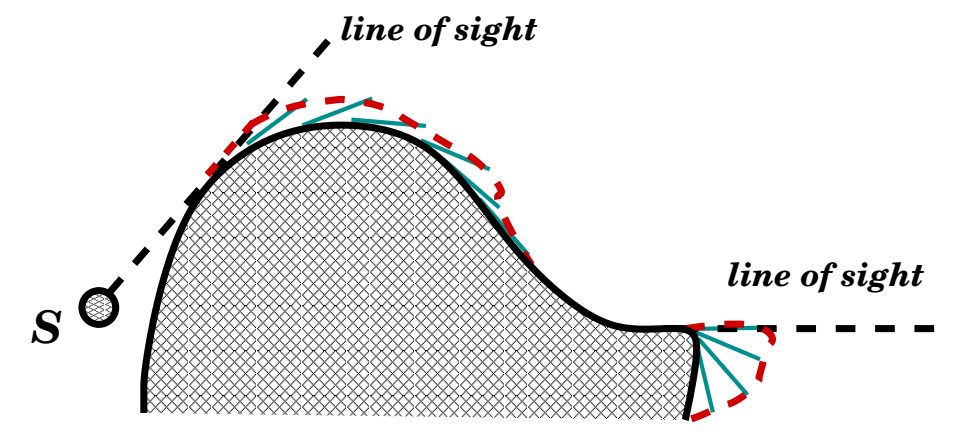

Figure 6: A demonstration of the leading order geometry of the dead-zone for a general obstacle with a point lighting source $S$. The critical radius (as the length of a tangential line segment with one end moving along the boundary of the obstacle) may have been exaggerated.

value (it corresponds to a failure curvature) we may conclude that a dead-zone exists and is attached to the boundary of the confinement.

\section{NUMERICAL TESTS WITH A MARKED PAR- TICLE DSD FRONT-TRACKER}

We use a marked particle front tracker for this purpose. The detonation front at a time step is presented by particles distributed on the front. A particle shall move in the normal direction with a velocity $D_{n}$ computed from a given $D_{n} \kappa$ relation. The front curvature $\kappa$ is obtained from fitting a curved surface to neighbor particles. For a boundary particle, the fitting is constrained by a material specific angle between the detonation front and the boundary condition. For example, in two dimensions, each internal particle carries two neighbor particles, one on each side, a circle fit to three points gives the curvature for the particle in middle. For a boundary particle, its two nearest neighbor particles are employed to fit a circle that intersect the wall with the angle $\omega_{c}$. If the boundary particle is ahead of the intersection of the fitting circle and the boundary, move the particle back to the intersection (fig. 7).

This method seems to be an easy one to implement, especially in two-dimensions for the particles are well ordered by arc-length of the front. However, there are a few issues regarding the front turning an obstacle associated with the methodology. First of all, because every interior particle moves in the normal direction of the front, 


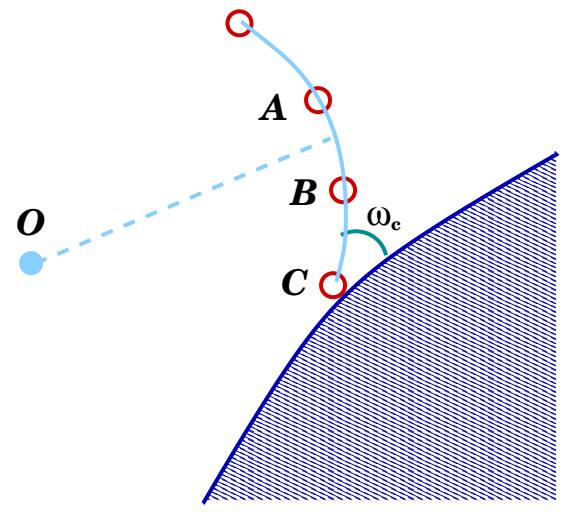

Figure 7: The contacting point $C$ between the detonation front and the HE boundary is obtained by fitting the two interior particles $A, B$ that are closest to the boundary with a circle (centered at $O$ ) that intersects the boundary with a given angle $\omega_{c}$. If the boundary particle is ahead of $C$, put it back to $C$.

so is not able to turn an obstacle (fig. 8, left); secondly, particles may run out of the boundary; thirdly, the arc-length and curvature of the front may change to cause resolution loss. The resolve the above issues of some particles run out of boundary, the fitting circle of the particle that are closest to the boundary, with its neighbor particles (one of them just becomes exterior) is used to intersect the boundary for the position of a boundary particle. The front is then reset after a time advancement of the front, by redistributing the particles on the front (fig 8, right).

\section{TEST 1: A RATE-STICK}

The rate-stick test maybe the simplest experiment to conduct for measuring a detonational front. With a rate stick test, a detonation wave propagates in a HE region bounded between two parallel straight lines with the distance between them being $2 R$. In an axi-symmetrical geometry, $R$ is the radius of a cylinder.

\section{AN ANALYTICAL INTEGRAL FOR A LINEAR $D_{n}-\kappa$}

The rate-stick problem can be scaled by $R$ and the coordinate system $(x, y)$ can be set such that the $x$-axis is the axis of symmetry. Therefore the boundary of the 

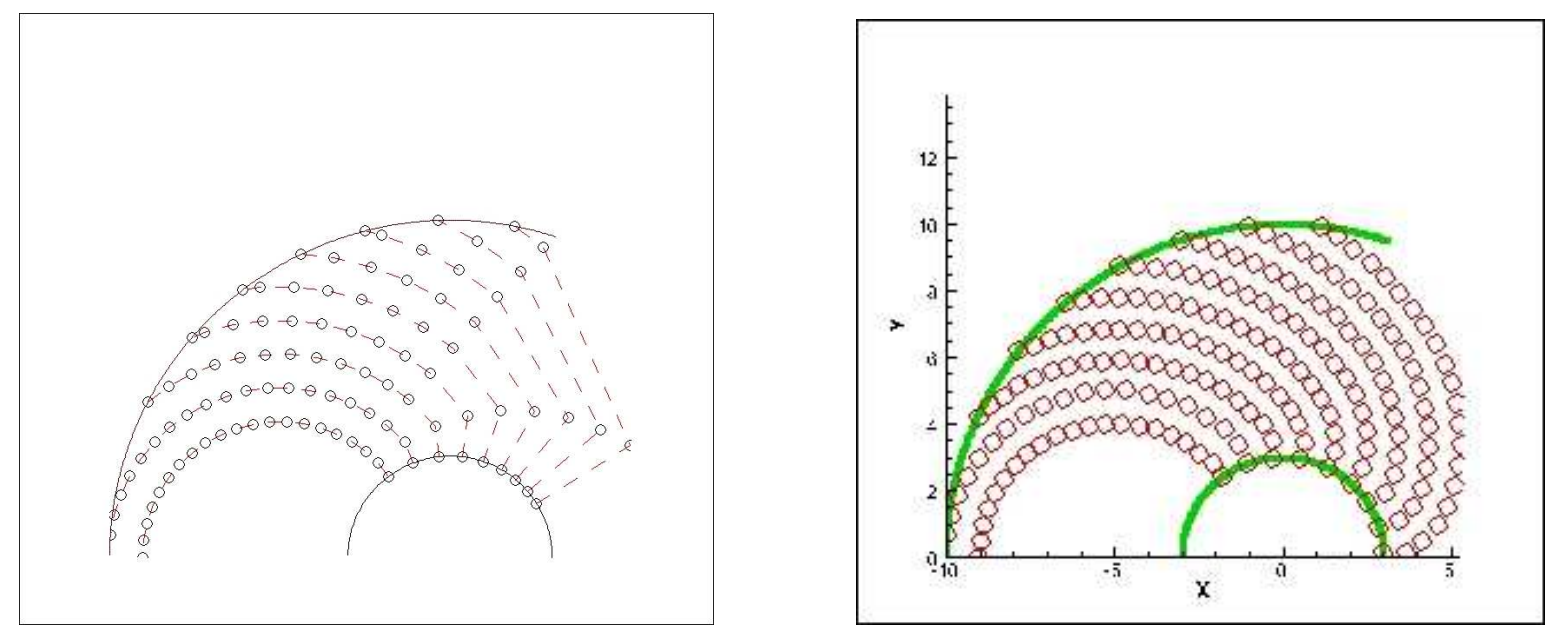

Figure 8: The marked particles in figure on the left are not relaxed tangentially thus some particles run out of boundary and most of the interior particles do not make the turn over the obstacle. IN the figure on the right, the front is reset by redistributing the particles on the front for keeping accuracy.

rate stick is $y= \pm 1$ in two dimensions and $r=1$ in the axi-symmetrical geometry. The direction of front propagation is the positive $\hat{\imath}$ direction.

To check the marked particle DSD-tracker we have, a linear $D_{n}-\kappa$ dependence that $D_{n}=D_{C J}-\alpha \kappa$ is employed because it has an analytical first integral in the two-dimensional planar geometry.

In order to derive the analytical integral, the equation of front motion $\phi_{t}+$ $D_{n}|\vec{\nabla} \phi|=0{ }^{[3]}$ can be written as

$$
f_{t}-D_{n} \sqrt{1+f_{y}^{2}}=0
$$

notice that at the intersection of front and the boundary $1 / \sqrt{1+f_{y}^{2}}=\sin (\omega)$ where $\omega$ in the angle of intersection. Thus one obtains

$$
D_{n}=\sin (\omega) f_{t}
$$

on boundary.

The governing equation can be written again as

$$
f_{t}=D_{C J} \sqrt{1+f_{y}^{2}}+\alpha \frac{f_{y y}}{\left(1+f_{y}^{2}\right)},
$$


for $\kappa=\vec{\nabla} \cdot \hat{n}=f_{y y} /\left(1+f_{y}^{2}\right)^{3 / 2}$, here that unit normal vector is defined by

$$
\hat{n}=\frac{\vec{\nabla} \phi}{\left|1+f_{y}^{2}\right|}=\frac{\hat{\imath}-f_{y} \hat{\jmath}}{\sqrt{1+f_{y}^{2}}}
$$

The speed of the traveling front $C$ can be obtained analytically in this case of a linear PDE. Let letter $s$ stand for the slope $f_{y}$, it is evident that $f_{y y}=d s / d y$, and the system governing the traveling front becomes

$$
\frac{d y}{d s}=\frac{\alpha}{C\left(1+s^{2}\right)-D_{C J}\left(1+s^{2}\right)^{3 / 2}},
$$

and an explicit definite integral exists that the width of the channel $2 R$ should be equal to

$$
\int_{S}^{-S} \frac{\alpha}{C\left(1+s^{2}\right)-D_{C J}\left(1+s^{2}\right)^{3 / 2}} d s=\frac{2 \alpha}{C \sqrt{D_{C J}^{2}-C^{2}}}\left(T_{1}+T_{2}+T_{3}\right),
$$

where $S$ is the slope at boundary with a value of $\tan \left(\omega_{c}\right)$.

$$
\begin{gathered}
T_{1}=-\sqrt{D_{C J}^{2}-C^{2}} \arctan (S), \\
T_{2}=D_{C J} \arctan \left(\frac{S D_{C J}}{\sqrt{D_{C J}^{2}-C^{2}}}\right), \\
T_{3}=D_{C J} \arctan \left(\frac{C S}{\sqrt{\left(1+S^{2}\right)\left(1-C^{2}\right)}}\right) .
\end{gathered}
$$

We clearly see that at the limit $\alpha \rightarrow 0$, as there is no curvature effect on the front normal velocity, the traveling wave speed must be equal to $D_{C J}$.

With our choice of $R=1$ (scaling in space), $D_{C J}=1$ (scaling in time), and $S=1$ (which means the angle between front and the boundary is kept to $\pi / 4$, the above integral is simplified to

$$
\frac{2 \alpha}{C \sqrt{1-C^{2}}}\left(-\frac{\pi \sqrt{1-C^{2}}}{4}+\arctan \left(\frac{1}{\sqrt{1-C^{2}}}\right)+\arctan \left(\frac{C}{\sqrt{2\left(D_{C J}^{2}-C^{2}\right)}}\right)\right)
$$


which should be equal to 2 , the width of the channel. The speed of the travelling wave, $C$, is now a function of $\alpha$. With the choice $\alpha=0.1$ and solve the above equation with an iteration method, one obtains that $C=0.969723$.

Run the marked particle DSD-tracker to time $=20$, with $d t=0.0001$ one obtains the following values of the traveling velocity, varying the number of particles on the detonation front. Because the time step need to be inversely proportional to the square of the space between neighbor particles ${ }^{[3]}$, we have not tried a finer resolution. The initial front geometry is a circle that intersects the boundary with $S=1$ to satisfy the DSD boundary angle condition. However, other rather arbitrary initial front geometries have been tried also and the same steady state of the traveling wave are obtained.

\begin{tabular}{|c|c|c|}
\hline$N$ & $v$ & $d v$ \\
\hline 101 & 0.969806 & $8.3 \times 10^{-5}$ \\
\hline 201 & 0.969744 & $2.1 \times 10^{-5}$ \\
\hline 401 & 0.969728 & $0.5 \times 10^{-5}$ \\
\hline
\end{tabular}

Table 1: The traveling velocity in a rate stick obtained with the marked particle DSDtracker for various numbers of particles distributed on the detonation front. $N$ is the number of particles distributed on the front, $v$ is the traveling velocity obtained with the DSD-tracker, and $d v$ is the difference to the theoretical value of the travelling velocity (for $\left.D_{n}=1-0.1 \kappa\right)$.

From the table above we can see a second order convergence with our DSDtracker. This numerical experiment verifies that the DSD-tracker obtains the correct steady state of a detonation. Figure 9 shows how a V-shaped initial front profile progresses toward the steady state with the DSD-tracker.

\section{TEST 2: WITH A CIRCULAR OBSTACLE}

The geometry of the problem is taken from the TP-03 problem reported in ${ }^{[15]}$. The boundary of $\mathrm{HE}$ is define by the arcs of a pair of concentric circles. The inner boundary has a radius of 3 and the outer boundary has a radius of 10 . Two initial front geometries has been tested. The first is a circle centered at $(-5,0)$, the second is a straight line segment. As our choices, both the initial front geometries intersect the inner boundary with a specified DSD angle of choice $\omega_{c}=\pi / 3$. This angle constraint is not applied to the outer boundary because the intersection angle between front 


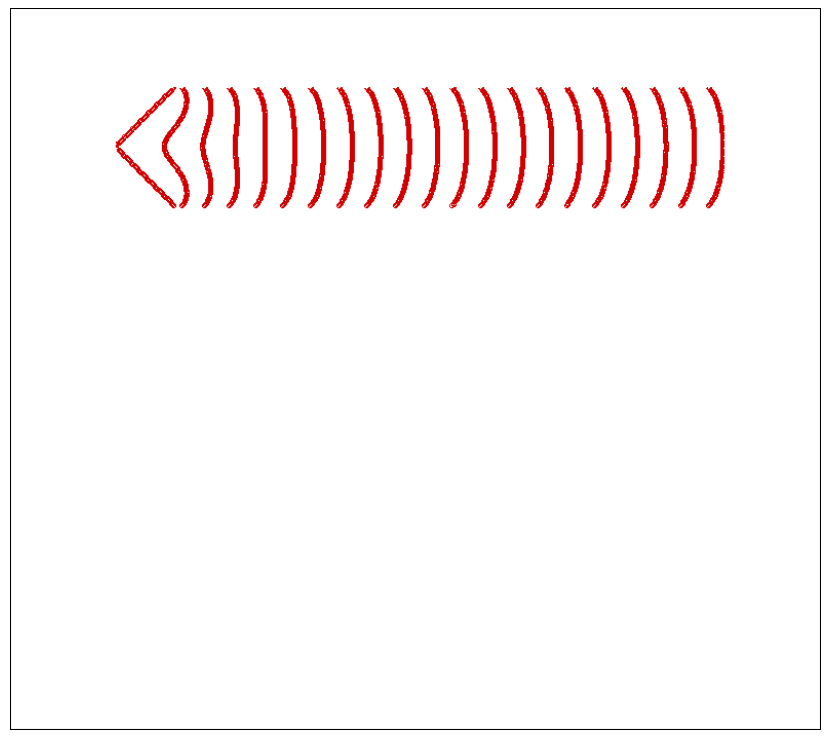

Figure 9: The steady state is obtained from a $V$-shaped initial front with the DSDtracker.

and boundary is always below $\omega_{c}$. A linear $D_{n}-\kappa$ relation is employed for the test in order to test the effect of the slope $d\left(D_{n}\right) / d(\kappa)$ on the terminal velocity of front at the boundary.

\section{The Detonation Fails on Inner Boundary}

The steady state for a "traveling wave" is arrived with both initial front geometry (fig. 10). Because the steady front shape is invariant to the initial front geometry, we compute the terminal velocity for a steady traveling detonation wave, varying the slope on the $D_{n}-\kappa$. The results are shown in fig. 11. If we consider a detonation would fail at $D_{n}=D_{f}<0.9 D_{C J}$, then each of the terminal velocity obtained from our DSD-tracker is below $D_{f}$. Therefore, there must be a layer of HE material that is not detonated, attached to the inner boundary. This is consistent with the conclusion obtained with the analytical solution for a Huygens front, that a general dead-zone must exist when a detonation turns over an obstacle, even when its surface is smooth. 


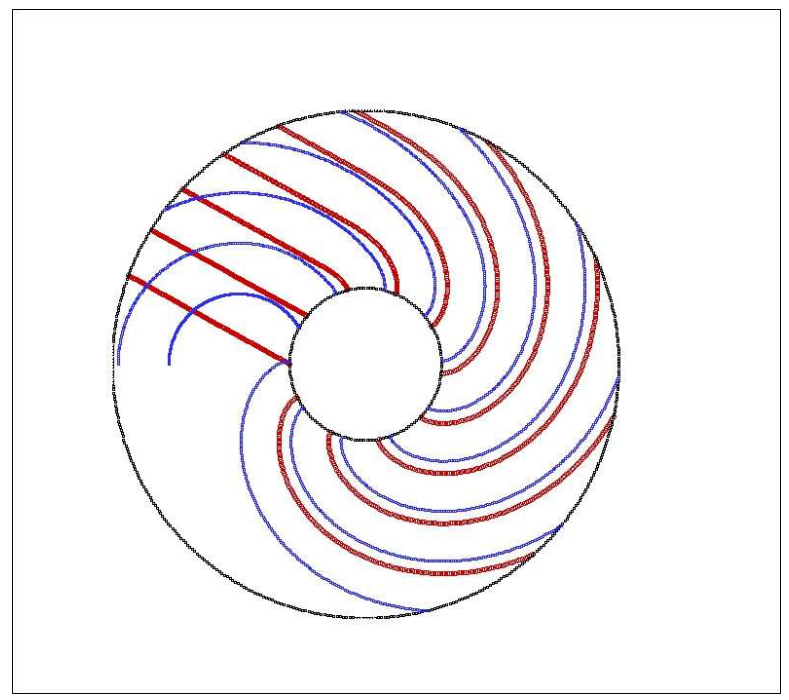

Figure 10: The same steady state is arrived for a circular channel with two different initial detonation fronts.

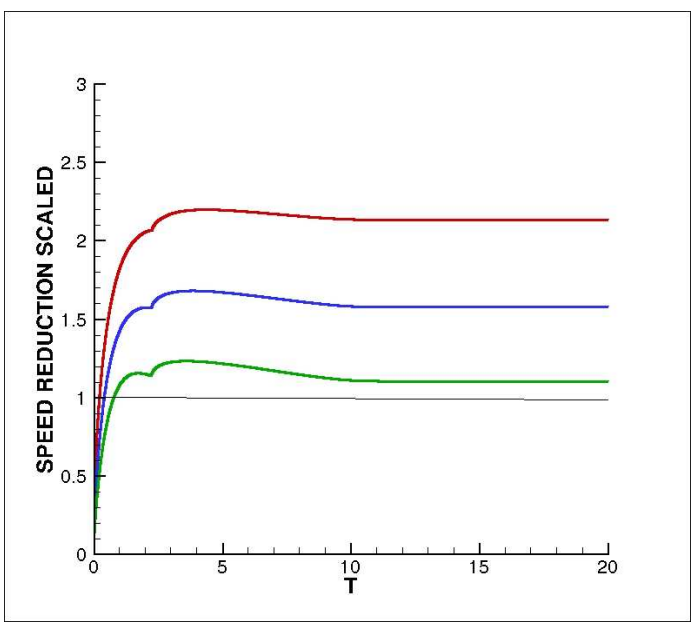

Figure 11: With a DSD boundary angle of $\pi / 3$, and a linear $D_{n}-\kappa$ that $D_{n}=1-\alpha \kappa$, the terminal velocity reduction from the CJ value on the boundary of a obstacle exceeds the failure value $\left(0.9 D_{C J}\right.$, marked by the line of height 1$)$ for $\alpha=0.05$ (top), $\alpha=0.025$ (middle), and $\alpha=0.0125$ for the circular test. 


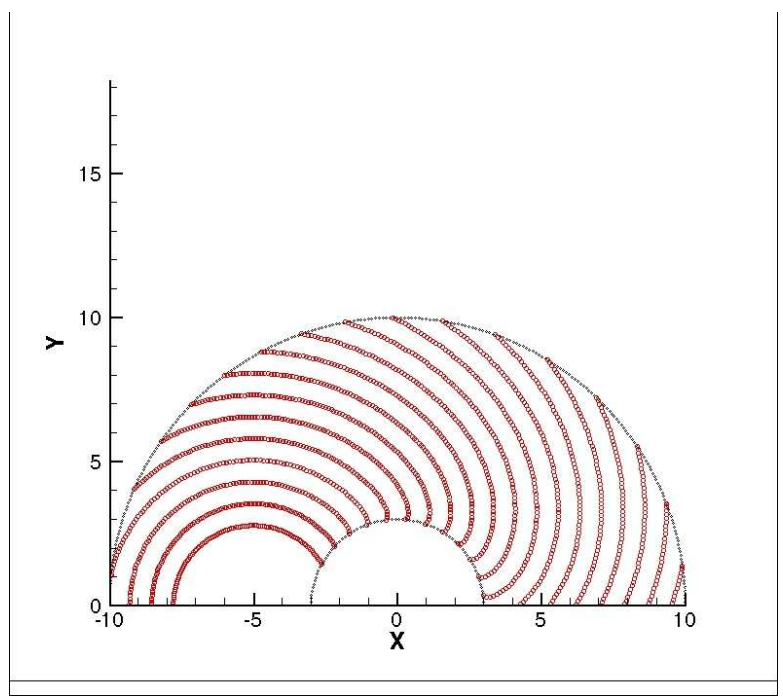

Figure 12: The lighting time contours for a PBX-9502 point detonation with the Circular geometry, obtained with our marked particle DSD-tracker. The boundary $D S D$ angle is set to $\pi / 4$.

\section{THE BURN-TABLE FOR PBX-9502}

For the HE material PBX-9052, its DSD properties have been obtained with nu-

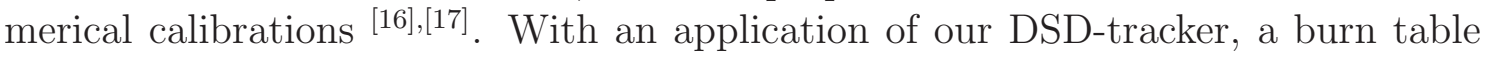
for two-dimensional geometry can be obtained for relistic simulations of material interaction with PBX-9502.

We employ the $D_{n}-\kappa$ relation provided in ${ }^{[17]}$ that

$$
\frac{D_{n}}{D_{C J}}=1+A\left[\left(\kappa_{c}-\kappa\right)^{\mu}-\kappa^{\mu}\right]-\frac{B \kappa^{\nu}}{1+C \kappa^{\lambda}},
$$

with $D_{C J}=7.818 \mathrm{~mm} / \mu \mathrm{s}$, the critical (failure) curvature $\kappa_{c}=1.276 \mathrm{~mm}^{-1}, A=$ $0.2643, B=0.5264, C=27.81$, and $\mu=0.8042, \nu=0.5264, \lambda=1.279$. The DSD boundary angle is set to $\omega_{c}=\pi / 4$, for PBX-9502.

For the Circular problem we run the marked particle DSD-tracker and obtain the detonation time contours shown in fig. 12. Fig. 13 show the DSD boundary angle is enforced on the inner boundary. 


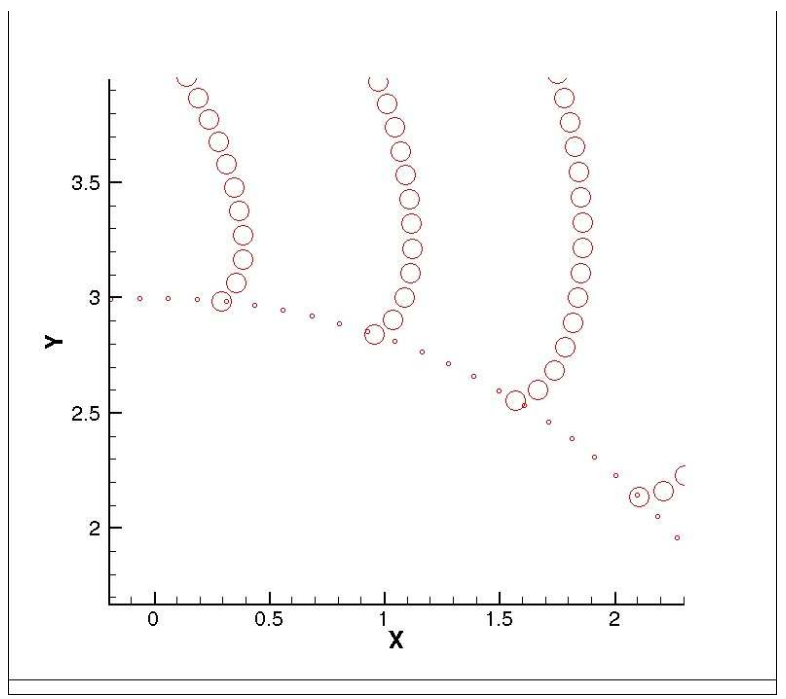

Figure 13: A close look of the DSD angle between detonation front and the boundary of an obstacle, with lighting time contours for a PBX-9502 point detonation in the Circular geometry, obtained with our marked particle DSD-tracker.

\section{CONVERTING THE BURN-TABLE TO LIGHTING TIME ON A MESH}

The lighting time contours, with marked particles distributed on each of them, provide the lighting time data points. For a hydro simulation the lighting time on a mesh is needed. For each HE node a lighting time needs be assigned from the data on marked particles. This is achieved by putting the nodes and the marked particles into a back ground virtual cubical. For a given node and the virtual cube contains it, one collects a list of marked particles that are also contained in this cube (fig. 14). The front arrival time on each particle of the list is used for a quadratic least-squared fit that minimized the target function

$$
J=\frac{1}{2} \sum_{1}^{n}\left(T_{l}(x, y)-T_{i}\right)^{2},
$$

where the lighting time is assumed to have the form

$$
T_{l}=a x^{2}+b y^{2}+c x y+d x+e y+f,
$$

where $n$ is the number of particles available to support the least-squared fitting $a, b, c, d, e$ and $f$ are parameters to be found by performing the fitting. 


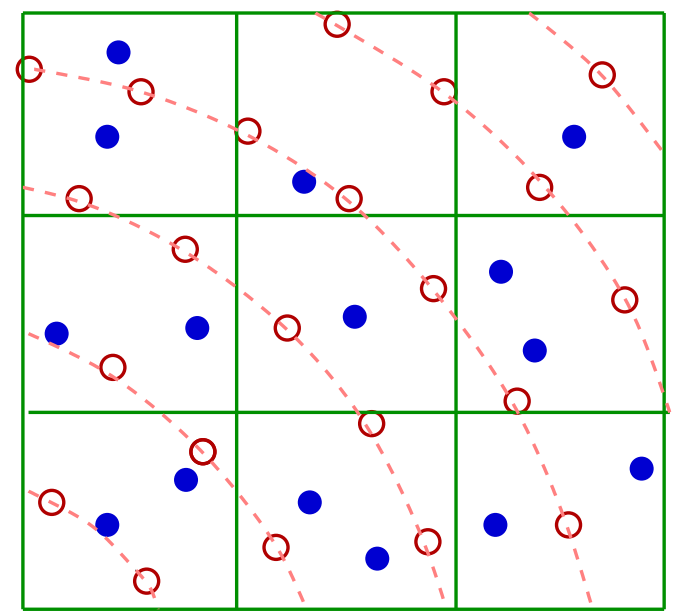

Figure 14: The red data points (marked particles on various time contours) as well as a given set of points (blue dots, considered as mesh nodes) are contained in a background virtual Cartesian mesh (the green grid). The lighting contours are the dash pink curves. The lighting time at a blue dot is obtained with a local least-squared quadratic fitting with the data carried by the marked particles contained in nearby virtual cubes. 
If not enough marked particles are there in the cube that contains the node, particles contained in neighbor cubes can be used for more data points to support the least squared solution. Clearly this approach would work for an arbitrarily distributed point set, thus the lighting time on an arbitrary hydro mesh can be obtained with running the DSD-tracker.

The lighting time on a hydro mesh for the circular geometry is obtained with the approach described above and is shown in fig. 15.

\section{CONCLUSION}

With the DSD (detonation shock dynamics) theory, a general dead-zone is described for a detonation front traveling over an obstacle and making turns continuously on the boundary of the obstacle. When the radius of curvature $|R|$ of the boundary of obstacle is zero, i.e. the case of a corner turning, the dimension of the dead-zone is predicted to be the same as the DSD critical radius $R_{c}=\kappa_{\max }^{-1}$ from the analysis in this report. In the case of a smooth obstacle $R_{c} /|R|=\epsilon \ll 1$, the thickness of the dead-zone is $\epsilon R_{c} / 2$. An experiment that varies the curvature of the obstacle should show a dead-zone gradually shrinking from the size of the critical radius (in the case of a corner turning) to a thin region as the curvature of the boundary of the obstacle reduces. This is in agreement with the fact that results form DNS show the reaction zone stays away from the HE boundary [16]. The analysis in the paper suggests that the DSD critical curvature $\kappa_{\max }$ for a certain HE material can be obtained from a corner turning test.

A marked particle DSD-tracker is used to verify that a general dead-zone may also exist for a DSD wave with a specified boundary angle between the detonation front and the boundary of the obstacle. This seems to be able to explain the fact that in a DNS calculation of reactive flow, the reaction region is detached from the $\mathrm{HE}$ confinement. The DSD-tracker has a second order convergence and this is verified with an analytical integral for a linear $D_{n}-\kappa$ relation. This DSD-racker can be used to obtain a burn table for two-dimensional or three-dimensional axis-symmetrical geometry, for a practical HE material such as PBX-9502. A method to convert the burn table data to lighting time on a hydro mesh is described and demonstrated with a PBX-9502 detonation for a curved geometry in two-dimensions. 


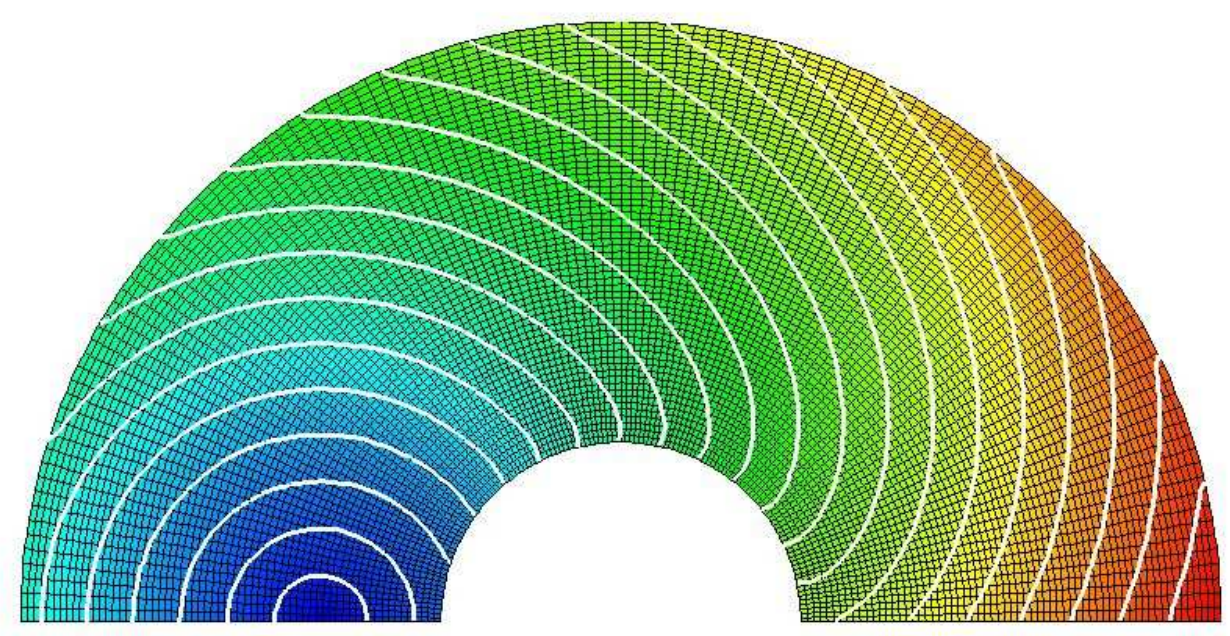

Figure 15: The burn table data at the marked particles on the countours for various time is converted to the lighting time on the nodes of a hydro mesh, with a local least-squared quadratic interpolation, for the circular test. 


\section{ACKNOWLEGDMENT}

The author would like to thank Dr. Al Nichols, Dr. Larry Fried, and Dr. Clark Souers at Lawrence Livermore National Laboratory for reviewing the paper. The author also appreciates communications with Dr. Tariq, Aslam at Los Alamos National Liboratory regarding his work with the $D_{n}-\kappa$ relation for PBX-9502.

\section{REFERENCE}

[1]. Detonation Wave Curvature, Corner Turning, and Unreacted Hugoniut of PBXN-111, J. W. Forbes, E.R. Lemar, G.T. Sutherland, and R.N. Baker, NSWCDD/ TR-92/164, Naval Surface Warfare Center, 1992.

[2]. On the Normal Detonation Shock Velocity-Curvature Relationship for Materials with Large Activation Energy, J. Yao, and D. S. Stewart, Combustion and Flame, 100, 519-528, 1995.

[3]. Level-Set Techniques Applied to Modeling Detonation Shock Dynamics, T. Aslam, J. Bdzil, and D. S. Stewart, Journal of Computational Physics, 126, 390409, 1996.

[4]. On the Dynamics of Multi-dimensional Detonation, J. Yao, and D. S. Stewart, Journal of Fluid Mechanics, 309, 225-275, 1996.

[5]. The Normal Detonation Shock Velocity-Curvature Relationship for Materials with Non-ideal Equation of State and Multiple Turning Points, Combustion and Flame, 113, 224-235, 1998.

[6]. Level Set Methods and Fast Marching Methods, J.A. Sethian, Cambridge University Press, 1999.

[7]. Proton Radiography and Numerical Modeling of colliding, Diverging PBX-9002 Detonations, C.L. Mader, J.D. Zumbro, and E. N. Ferm, Proceedings of the $12^{\text {th }}$ International Detonation Symposium, 2002.

[8]. Reactive Flow Modeling of the Interaction of TATB Detonation Waves with Inert Materials, C. M. Tarver and E. M. Mcguire, Proceedings of the $12^{\text {th }}$ International Detonation Symposium, 2002.

[9]. LX-17 Corner-Turning and Reactive Flow Failure, P. C. Souers, H. G. Andreski, C.F. Cook III, R. Garza, R. Pastrone, D. Phillips, F. Roeske, P. Vitello and J.D. Molitoris, Propellants, Explosives, Pyrotechnics, Vol. 29, 6, pp.359-367, 2004. 
[10]. Proton Radiography Examination of Unburned Regions in PBX-9502 Corner Turning Experiments, E. N. Ferm,. C. L. Morris, J. P. Quintana, P. Panzuchanic, H. Stacy, J.D. Zumbro, G. Hogan, and N. King, Proceedings of Shock Compression of Condensed Matter, 2001.

[11]. A Validation of First-Order Detonation Shock Dynamics Theory, D. Lambert, S. Yoo, and D.S. Stewart, Proceedings of the Winter Simulation Conference, 2005.

[12]. A Geometrical DSD Lighting Algorithm on Arbitrary Unstructured Meshes, J. Yao, UCRL-JRNL-213932, Lawrence Livermore National Laboratory, 2005.

[13]. Detonation Waves in PBX 9501, R. Menikoff, Combustion Theory and Modeling, Vol. 10, 6, 1003-1021, 2006.

[14]. Dead Zones in LX-17 and PBX 9502, P. C. Souers, H. G. Andreski, (and others), Propellants, Explosives, Pyrotechnics, Vol. 31, 2, pp.89-97, 2006.

[15]. HE Burn Table Verification Problem, M.A. Kenamond, LANL Technique Report LA-UR 11-03096, 2011.

[16]. Front Curvature Rate Stick Measurements and Detonational Shock Dynamics Calibration for PBX-9502 Over a Wide Temperature Range, L. G. Hill, J. B. Bdzil, and T. D. Aslam, Eleventh International Detonation Symposium, 1998

[17] Extensions to DSD Theory: Analysis of PBX 9502 Rate Stick Data, T. D. Aslam, J. B. Bdzil and L. G. Hill, Eleventh International Detonation Symposium, 1998.

[18]. Test Problems for DSD2D, John B. Bdzil, Rudolph J. Henninger, and John, W. Walter, LA-14277, 2006. 